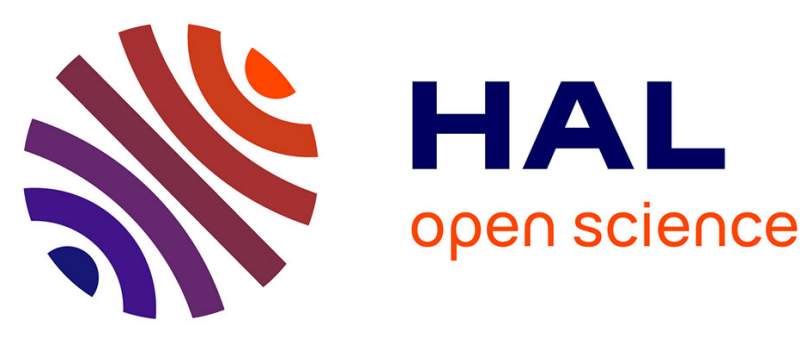

\title{
Factives and Intensionality
}

Richard Zuber

\section{To cite this version:}

Richard Zuber. Factives and Intensionality. New Frontiers in Artificial Intelligence JSAI-isAI 2010

Workshops, LENLS, JURISIN, AMBN, ISS, 2011, Tokyo, Japan. pp.104-114. halshs-00751202

\section{HAL Id: halshs-00751202 \\ https://shs.hal.science/halshs-00751202}

Submitted on 15 Nov 2012

HAL is a multi-disciplinary open access archive for the deposit and dissemination of scientific research documents, whether they are published or not. The documents may come from teaching and research institutions in France or abroad, or from public or private research centers.
L'archive ouverte pluridisciplinaire HAL, est destinée au dépôt et à la diffusion de documents scientifiques de niveau recherche, publiés ou non, émanant des établissements d'enseignement et de recherche français ou étrangers, des laboratoires publics ou privés. 


\title{
Factives and Intensionality
}

\author{
Richard Zuber \\ Rayé des cadres du CNRS, Paris, France \\ Richard.Zuber@linguist.jussieu.fr
}

\begin{abstract}
Notion of normal intensionality and of intensionality preserving negation are introduced. Both these notions apply to intensional sentential operators. When applied to factive operators they allows us to understand the way these operators give rise to presuppositions and their duals, assertions. They allow us also to distinguish semantically emotive factives from non-emotive ones.
\end{abstract}

\section{Introduction}

Factives are verbs of propositional attitudes. As is well-known verbs of propositional attitudes and operators formed from them are traditionally considered as posing special problems in formal semantics of natural languages on the one hand and as giving rise to particular questions in the philosophy of language and of knowledge. The difficulties that formal semantician encounters when approaching these verbs and constructions in which they occur are usually related to the fact that these verbs form intensional contexts and thus violate in various ways some general principles accepted in formal semantics (Partee 1979). In particular their strong intensionality, roughly speaking is not easily compatible with a compositional treatment of their semantics (Pelletier 1994)). In this paper I want to discuss something which, as far as I can tell, not has been explicitly discussed in the context of verbs of propositional attitudes : these are, roughly speaking, specific entailment like relations which hold between sentences (expressions) formed from verbs of propositional attitudes or their arguments and which are caused precisely by the intensionality of these verbs. Thus I will discuss the well-known relation of presupposition of factive verbs and the less discussed, but related relation of assertion. As we will see both these relations are easily obtainable in the context of some sub-set of verbs of propositional attitudes and a specific but natural negation of them.

Two warnings are needed at the outset. First, though I will discuss some semantic properties of sentential operators formed from verbs of propositional attitudes, I will not, strictly speaking discuss the semantics of these verbs themselves. Their semantics has many sides and probably is not separable from various pragmatic aspects. I will discuss those semantic aspects of sentential operators formed from verbs of propositional attitudes which are essential for presuppositions and assertions which can be associated with them. this means in particular that I will treat such operators as syntactically and semantically indecomposable wholes. Thus I will mostly ignore the fact that such verbs take grammatical 
subjects usually referring to human beings. I will also ignore the fact that such verbs can take quantificational noun phrases as grammatical subjects. This does not seem to be harmful since usually the subject position is considered as not giving rise to intensionality. Consequently I will only analyse sentential operators of the form $A V$ that where $A$ is a proper name and $\mathrm{V}$ a verb of propositional attitudes.

The second warning concerns the notion of presupposition. I want to stress that this paper does not offer any general theory of presuppositions. There is a huge literature concerning presuppositions both from theoretical and empirical points of view. An important topic in these considerations concerns the problem of presupposition projection that is the way presuppositions of more complex expressions are obtained from presuppositions of their simpler components. Again, this will not be considered here. Presuppositions that we will discuss are presuppositions of factives only. As will be seen, the way the existence of presuppositions of factives is explained does not easily generalise to other presupposing constructions. The reason is that the notion of normal intensional negation which plays essential role in the understanding of presuppositions of factives does not apply to cases in which intensionality is not involved.

As indicated above, I will discuss various entailments between sentences formed from sentential operators (which are formed from the verbs of propositional attitudes). The notion of entailment is taken in rather an intuitive sense. It takes for granted the notion of a possible world. Thus simply sentence $S$ is said to entail a sentence $T$ iff $T$ is true in every possible world in which $S$ is true. No more sophisticated notion of a logical model will be used.

There are at least three cases of entailments, or more generally of implications, under intensional operators interesting from the semantic point of view. The first is the case of factives predicates presupposing their sentential complements, the second is the entailment between two similar sentences with the same factive predicate in both sentences and differing only by the complementizer (that vs whether) of the factive. Finally there is a case of "neg-transportation" entailments (implications) to which some intensional predicates give rise. I will only mention this third type of entailment since it does not involve factive verbs. In the next section I recall more specifically these three cases with particular intention paid to the first two types involving factive verbs. Then in the next section I propose a specific notion of negation applicable to verbs of propositional attitudes which allows us to understand both types of entailments to which factives give rise.

\section{Factives}

Since the publication of Kiparsky and Kiparsky 1970) the term factive refers in linguistic semantics to verbs and predicates like to know, to forget, to regret, be sad, be strange, etc. when they take sentential complements. These predicates have an interesting semantic property : when completed by the grammatical subject referring to a human (which is usually the most natural case for them) they 
form sentential operators which presuppose the truth of the sentential complement that they take as argument. More specifically (positive forms of) sentences formed from factive predicates and their (natural) negations both entail the complement sentence. Thus (1a) and (1b)

(1a) Leo knows/regrets that $\mathrm{P}$

(1b) Leo does not know/not regret that $\mathrm{P}$

both seem to entail, on the most natural and generally accepted reading, that the sentence $\mathrm{P}$ is true.

Although before Kiparsky's article some philosophers also have tried to analyse certain verbs taking sentential complements with the help of the notion of presupposition (cf. Filmore 1963, Odegard 1965), their work was much more complete, empirically rich and gave rise to the renewal of the research on presuppositions. Indeed, sentences with factives represented a new set of presupposing constructions, different from those traditionally studied in the context of definite descriptions for instance. These new constructions showed that, as in the case of existential presuppositions, the notion of negation needed in the definition of presupposition must be different from the classical negation. This is because, as often has been pointed out, the sentence Leo does not know that $P$ can be considered as true in the case when $\mathrm{P}$ is false (cf. Leo does not know that $P$ because not- $P$ ).

Kiparsky's distinguish in part by syntactic means, two type of factive predicates: non-emotive and emotive ones. The first class includes verbs like know, remember, realise and to emotive factives belong verbs like regret, resent or predicates like be sad, be strange, be interesting etc, when they take impersonal subjects.

It is not clear to what extent the syntax alone is sufficient to distinguish clearly emotive from non-emotive factive predicates. Some of criteria for such a distinction seem to be language dependent. Let me mention here the differences which are important for the purposes of this article. One difference between those two classes is that, informally, emotive factives seem to be sensitive to more, contingent and non-contingent, sentences than non-emotive ones. Consequently emotive factives presuppose "more" than non-emotive ones. Consider the following examples:

(2a) Leo knows that the bottle is half empty.

(2b) Leo knows that the bottle is half full.

(3a) Leo regrets that the bottle is half empty.

(3b) Leo regrets that the bottle is half full.

Very likely sentences in (2) have both the same truth value whereas those in (3) have different truth values. We express this by saying that Leo regrets that is intensionally stronger than Leo knows that. The notion of the "strength of intensionality" will be made more precise in what follows. 
The second difference between emotive and non-emotive factives is that emotive, but not non-emotive, seem to presuppose in addition "knowledge" of the truth of the complement sentence (Zuber 1977). For instance (4a) presupposes (4b) and (5a) presupposes (5b) :

(4a) Leo regrets that $\mathrm{P}$.

(4b) Leo knows that P.

(5a) It is strange that $\mathrm{P}$.

(5b) It is known that $\mathrm{P}$.

Obviously sentences with non-emotive factives do not have such a presupposition. For instance (4b) does not presuppose itself nor (5b). Neither do these sentences presuppose a belief of any kind by the human beings denoted by the subject noun phrases occurring in them.

Finally, non-emotive factives differ from emotive ones by the fact that only non-emotive can take in addition the complementizer whether. Thus we have know whether and remember whether but not ${ }^{*}$ regret whether or ${ }^{*}$ it is sad whether.

The fact that intensional verbs forming non-emotive factives can take two complementizers (that vs whether) can be related to another semantic relation to which non-emotive factives give rise. This relation, which can be called assertion (in opposition to presupposition). It can be illustrated by the following examples:

(6a) Leo knows that $\mathrm{P}$.

(6b) Leo knows whether P.

(7a) Leo does not know that P.

(7b) Leo does not know whether P.

As already has been indicated, it is generally accepted, also by logicians, that (6a) entails (6b). Although it has not been often claimed so, it is also true that (7a), taken as presupposing $\mathrm{P}$, entails (7b). Indeed, suppose a contrario that (7a) is true and (7b) is false. The falsity of (7b) means that either (1) Leo knows that $\mathrm{P}$ or (2) Leo knows that not-P. This is, however, impossible: (1) cannot be true because (7a) is true and (2) cannot be true because (7a) presupposes the truth of P. Notice that the negation in (7a) and (7b) cannot be interpreted by the classical propositional negation (obviously syntactically it is not) and in particular it must be a presupposition preserving negation in (7a). Thus (8a) can be true and $(8 \mathrm{~b})$ false in the situation in which Leo knows that not-P is true:

(8a) It is not true that Leo knows that $\mathrm{P}$.

(8b) Leo knows whether P.

The last case of intensional sentence-embedding predicates giving rise to specific entailments concerns the so-called Neg-raising predicates. As I said I will not 
discuss them in detail but I only indicate their main property. These predicates have the following property: when negated sentences with such predicates imply a corresponding sentence in which the negation takes scope in the embedded clause. Thus, intuitively (9a) implies (9b):

(9a) Leo does not think that life is sad.

(9b) Leo thinks that life is not sad.

Notice that the negation in (8a) cannot be interpreted as just the metalinguistic or classical propositional negation. Indeed it is easy to see that (10) does not imply (9b):

(10) It is not true that Leo thinks that life is sad.

For instance in most cases when Leo is sleeping (10) is true and (9b) is false.

Concerning the relation between $(9 \mathrm{a})$ and $(9 \mathrm{~b})$ one could also ask whether these two sentences are equivalent: should one consider that (9b) also implies (9a). We will not discuss this possibility since it does not concern factive operators.

We can thus see that in the all three cases of entailments (or implications) presented above we need a special negation. The purpose of this paper is to show that we have the same negation in the case of presupposition of factives and in the case of assertion of factives.

\section{Intensionality preserving negation}

Obviously entailments and implications concerning presuppositions, assertions and neg-transportation essentially involve a negation. Moreover the cases we are considering involve intensional functional expressions (intensional sentential operators). This means that defining the negation we have to take into account precisely the fact that it has to apply to intensional functional expressions. So first we have to precise the notion of intensionality of such expressions and then define the appropriate negation of them.

Intensionality of functional expressions that we will use is characterised by the fact that the Leibniz' law does not hold in their context. Since we are basically interested in intensional operators taking sentences as arguments, the failure of Leibniz' law just means that the substitution of sentential arguments by logically equivalent sentences does not preserve truth-value. Since we are interested in sentential operators formed from verbs of propositional attitudes which take as grammatical subjects NPs referring to human beings it is preferable to relativise he notion of intensionality to a possible world. More precisely we will adopt the following definition:

Definition 1. A sentential operator $O$ (an expression of the category $S / S$ ) is normally intensional iff for some sentence $P O(P)$ is true in some world $w$ and 
for every sentence $P$ and every possible world $w$, if $O(P)$ is true in $w$, then there exists a sentence $P^{\prime}$ such that $P$ and $P^{\prime}$ have the same truth-value in $w$ and $O\left(P^{\prime}\right)$ is false in $w$.

Few remarks about the definition. First, as already indicated, we will treat syntactically complex expressions consisting of a grammatical subject and a verb of propositional attitude as operators having syntactically non complex form. This maybe be a simplification. Second, as we will see, definition D1 does not make directly the difference between the intensionality of classical modal operators and the intensionality of operators formed from verbs of propositional attitudes. Indeed we know that modal operators display weaker intensionality than the propositional attitude verbs (Creswell 1975). In addition they do not give rise to semantic relations discussed above. In particular the necessity operator does not presuppose, but only entails, the truth of its argument. One could make the indicated difference by imposing the condition of contingency on sentences used in substitution; none of the sentences which substitute the other can be a necessary sentence. We will see that it is also possible to make the difference by the appropriate notion of negation of normally intensional operators.

Finally, it follows from definition, 1 that for a sentential operator to be normally intensional it must be possible to show that the appropriate substitution never preserves truth-value (and not only in some cases as it is the case for "classical" intensionality). This feature can be related to the fact that intensional operators we consider here are strictly speaking syntactically complex being composed of a subject NP and a verb phrase. The subject NP usually refers, directly or indirectly, to human beings, which are non omniscient and sensitive to different ways of presentation of the same semantic content. Different logically equivalent sentences can be considered as different ways of presentation of the same (logical) content.

One can check that the sentential operators formed with verbs of propositional attitudes (not only factive predicates) are normally intensional. This is true not only of predicates whose subject NPs refers to human beings but also of predicates whose subject NP is the impersonal pronoun it. The classical modal operator of necessity is also normally intensional. the possibility operator is not normally intensional.

Although we will not be interested in (Booleanly) complex intensional operators nor in the problem of calculating presuppositions and assertions of complex sentences notice the following simple facts concerning some compositions of normally intensional operators:

Proposition 1. Let $\mathrm{O}_{1}$ and $\mathrm{O}_{2}$ be two normally intensional operators such that $\mathrm{O}_{1} \neq \neg \mathrm{O}_{2}$ (where $\neg$ is the classical negation), $T$ the classical truth operator and $N$ the classical propositional negation. Then:

(i) $O_{1} \wedge O_{2}$ and $T \wedge O_{1}$ are normally intensional.

(ii) $O_{1} \vee O_{2}$ and $N \vee O_{1}$ are not normally intensional.

We want now to define a natural negation of intensional operators. We observe that some of such intensional operators can be considered as being related 
to other intensional operators by a specific lexical negation making them contrary to each other. This is the case for instance with believe that vs doubt that or remember that vs forget that. Notice that both members of these pairs are normally intensional. So we can suppose that there not only lexical but also a syntactic (and semantic) way to negate normally intensional operators. This can be done by the normal negation $n$ whose semantics is defined as follows:

Definition 2. Let $O$ be a normally intensional operator. Then $n-O$ is the normal negation of $O$ iff for any sentence $P, n-O(P)$ has the same truth value as $\neg(O(P))$ and $n-O$ is a normally intensional operator (where $\neg$ is the classical negation, and, since $O$ is of the category $S / S, n$ is of the category $(S / S) /(S / S)$ ).

Thus $n$ does the job of ordinary negation, but in addition it preserves the intensionality of the operator to which is applies.

Obviously normal intensional negation $n$ entails the "ordinary" extensional negation but not the other way around. In addition the ordinary negation $\neg$ does not preserve the normal intensionality. We can show this with the following example. Let $O$ be a factive operator Leo knows that (which is normally intensional) and consider the complex propositional operator $\neg O$ : It is not true that Leo knows that. Clearly, if $P$ is false $\neg O(P)$ is true. However since $P$ is false there is no sentence $P_{1}$ equivalent to $P$ such that $\neg O\left(P_{1}\right)$ would be false. Thus $\neg O$ is not normally intensional even though $O$ is.

Notice also that definition 2 does not guarantee that all normally intensional operators have a normal negation. For instance the modal operator of necessity does not have a normal negation.

The importance of the normal negation is shown in the following proposition which indicates that normal negation is precisely the negation which is used when factives give rise to presuppositions:

Proposition 2. If $O$ is a normally intensional operator such that for any $P$, $O(P)$ entails $P$, then $n-O(P)$ also entails $P$.

Proof. (Zuber 1980): Suppose a contrario that there exists a world $w$ such that $n-O(P)$ is true in $w$ and $P$ is false. This means, given definition 2, that there exists $P^{\prime}$ with the same truth value as $P$ in $w$ and such that $O\left(P^{\prime}\right)$ is true in $w$. But this is impossible since $O\left(P^{\prime}\right)$ entails $P^{\prime}$.

Thus factives are verbs of propositional attitudes which, roughly speaking, etail the sentential complement to which they apply. Consequently we are justified in calling factives verbs of propositional attitudes which entail their sentential argument because their normal negation also entails it.

As indicated there are two types of factives, emotive and non-emotive ones. Examples like (2) and (3) above, and like the following ones, suggest that emotive factives distinguish a "stronger semantic equivalence" than the equivalence distinguished by non-emotive factives:

(11a) Leo knows that Bill bought a car from Sam.

(11b) Leo knows that Sam sold a car to Bill. 
(12a) Leo regrets that Bill bought a car from Sam.

(12b) Leo regrets that Sam sold a car to Bill.

For our purposes we can assume, at least for some possible world $w$, that (11a) and (11b) have the same truth value (in $w$ ) whereas (12a) and (12b) differ in their truth value (in $w$ ). This supposition means that the emotive factive operators (Leo regrets that) are in some sense intensionally stronger than the non-emotive ones.

It follows from the above examples that $A$ regrets that is intensionally stronger than $A$ knows that in the sense that is is sensitive to more arguments. Similarly it has been noticed (Creswell 1975) that verbs of propositional attitude are more strongly intensional than for instance the classical modal operator of necessity. On way to show this difference is to point out that intensionality of verbs of propositional attitudes can be tested by a pair of materially equivalent sentences such that both members of the pair are contingent sentences. This is not the case with the operator of necessity: we can show that this operator is intensional only using a pair of equivalent sentences such that one of the sentences is contingent and the other one non-contingent (necessarily true). So our definition of the strength of intensionality has to take into account these facts. Furthermore we want also that if $O$ is normally intensional then $O$ and $n-O$ should have the same intensional strenght. These observations lead to a series of a new notions. First we define a detector of intensionality:

Definition 3. The set of two sentences $P, P^{\prime}$ is a detector of intensionality of the operator $O$ (in the world $w$ ), iff $P$ and $P^{\prime}$ have the same truth value in $w$, but $O(P)$ and $O\left(P^{\prime}\right)$ have different truth-values in $w$.

Obviously extensional propositional operators do not have detectors of intensionality (in no possible world). Furthermore, any detector of intensionality of the modal operator of necessity contains a necessarily true sentence as a member. Normally intensional operators formed from verbs of propositional attitudes can have detectors of intensionality which contain only sentences.

With the help of the detector of intensionality we define the notion of the degree of intensionality of a given sentential operator, which needs not to be normally intensional:

Definition 4. Degree of intensionality of the sentential operator $O$ in the possible world $w$, noted $D I(w, O)$, is the set of the detectors of intensionality of $O$ in $w$.

For instance the degree of intensionality of an extensional sentential operator (in any possible world) is the empty set.

We can now precise how to compare the intensional strength of two sentential operators:

Definition 5. The sentential operator $O$ is intensionally stronger than the intensional operator $O^{\prime}$ iff for any possible world $w$ the set $D I\left(w, O^{\prime}\right)$ is strictly 
included in the set $D I(w, O)$. If these sets are equal then $O$ and $O^{\prime}$ have the same intensional strength.

Notice that since the set-inclusion constitutes a partial order there are normally intensional operators which cannot be compared from the point of view of their strength of intensionality.

Concerning normally intensional operators and their normal negation the following fact is true:

Proposition 3. For any possible world $w$ and any normally intensional operator $O$ the following equality holds: $D I(w, O)=D I(w, n-O)$.

Thus normal intensional negation preserves the degree of intensionality of a given intensional operator. This means in particular that normal intensional negation is not intensional operator. In fact in some sense it is even "stronger" than the clasical extensional negation. This observation will be used when discussing some properties of assertions.

Definitions 2 and 5 allow us to prove the following proposition which accounts for the presuppositions of emotive factives (Zuber 1982):

Proposition 4. If $O$ is a sentential operator which is intensionally stronger than $O^{\prime}$ and for any $P, O(P)$ entails $O^{\prime}(P)$ then $n-O(P)$ entails $O^{\prime}(P)$.

Proof. Suppose a contrario that there exists a world $w$ such that $n-O(P)$ is true in $w$ and $O(P)$ is false (in $w$ ). Since $O$ is intensionally stronger than $O^{\prime}$ this means, given definition 5 and proposition 3 , that there exist sentences $P_{1}$ and $P_{2}$ which are detectors of intensionality of $O$ but are not detectors of intensionality of $O^{\prime}$ (in $w$ ). Consider now the following two sets of sentences $\left\{P, P_{1}\right\}$ and $\left.\} P, P_{2}\right\}$ as possible detectors of intensionality of $O$ and $O^{\prime}$ (in $w$ ). Suppose first that $\left\{P, P_{1}\right\} \in D I(O, w)$. It follows from this supposition that $\left\{P, P_{1}\right\} \notin D I\left(O^{\prime}, w\right)$ and thus that $O^{\prime}\left(P_{1}\right)$ is false in $w$. This is impossible, however, because $O\left(P_{1}\right)$ is true in $w$ (since $n-O(P)$ is true in $w$ ). Suppose now that $\left\{P, P_{1}\right\} \notin D I(O, w)$. It is easy to show that in this case $\left\{P, P_{2}\right\} \in D I(O, w)$ and $\left\{P, P_{2}\right\} \notin D I\left(O^{\prime}, w\right)$. But this is again impossible because in this case $O\left(P_{2}\right)$ would be true and $O^{\prime}\left(P_{2}\right)$ false in $w$. This a contrario supposition leads to a contradiction.

Notice that proposition 4 is a generalisation of proposition 2, if we suppose that any sentence $P$ is equivalent to $T(P)$, where $T$ is the classical truth operator. The reason is that $T$ can be considered, according to definition 5 as intensionally weaker than any normally intensional operator.

Concerning the relation of assertion one can show that it is related to the identical intensionality strength of sentential operators. More precisely the following proposition is true (Zuber 1982):

Proposition 5. If $O$ and $O^{\prime}$ have the same intensional strength and $O(P)$ entails $O\left(P^{\prime}\right)$ then $n-O(P)$ entails $n-O^{\prime}(P)$. 
Proof. Suppose a contrario that there exists a world $w$ such that $n-O(P)$ is true in $w$ and $n-O^{\prime}(P)$ is false (in $w$ ). Since $n-O$ is normally intensional there is a sentence $P^{\prime}$ which forms with $P$ a detector of intensionality of $O$ and $n-O$ in $w$. Given the hypothesis of the proposition the set $\left\{P, P_{1}\right\}$ is also the detector of intensionality of $O^{\prime}$ and $n-O^{\prime}$. But then $O\left(P_{1}\right)$ would be true and $O^{\prime}\left(P_{1}\right)$ false in $w$ which is impossible.

Proposition 5 can be illustrated by examples in (6) and (7): since (6a) entails (6b) and Leo knows that and Leo knows whether have the same intensional strength, we have to conclude, given proposition 5 , that $(7 \mathrm{a})$, the intensional negation of (6a) entails (7b), the intensional negation of (6b). Thus we can say, roughly, that know that asserts know whether. And this is true of many other non-emotive factives.

\section{Conclusions}

A negation preserving intensionality applying to verbs of propositional attitudes have been introduced in order to explain the mechanism producing in particular presuppositions of factive prdicates. Though the idea of such a negation is intuitivcely clear its precise rendering, I must say, is not obvious. It is quite possible that another non-equivalent definition of intensional negation, in which partial functions are used, is necessary.

The machinery proposed for the explication of factive presuppositions have also been used to justify semantically the distinction between emotive and nonemotive factives. It is well-know that there is not a general agfreement concerning the data to which give rise emotive factive. I considered, following Zuber 1977, that they presuppose knowledge. Some researches consdider that they presuppose only belief. Still some others conside that they are not even veridical (for the review of diferent stands see Egré 2008). It seems to me that one can reach the final conclusion in these matters after considering other syntactic and semantic factors (cf. Egré 2008, Zuber 1982).

The above analyses cannot be generalised to implicative verbs (Karttunen 1971). The reason is that they are not normally intensional (at the predicate level). More specifically it is not true that given an implicative verb (to manage, to forget, etc.) and a predicate to which it applies, one can always find another co-extensional predicate such that the substitution of the one predicate by the other one will (systematically) lead to the change of the extension of the whole complex (modified by the implicative verb) predicate. In fact it is often very difficult to decide whether different VPs have the same denotation. The reason is that that VPs with implicative modifiers probably denote events. Sharvit 2003 shows that the verb try lexically related to many implicative modifiers is not intensional (though not exactly in the sense used here). And indeed implicatives give rise to different implications than factives. This is in particular true with negated implicatives. 


\section{References}

Cresswell, M.: Hyperintensional logic, Studia Logica 34 (1975) 25-38

Egré, P.: Question-Embedding and Factivity. Philosophische Studien 77 (2008) 85-125

Fillmore, C.: Entailments rules in a semantic theory. Project on Linguistic Analysis Report N 10 (1965), reprinted in Rosenberg and Travis (eds.) Readings in the Philosophy of Language, Prentice Hall (1972)

Karttunen, L.: Implicative verbs. Language 47 (1971) 340-358

Kiparsky, P. and C. Kiparsky: Fact. in M. Bierwisch and K.E. Heidolph (eds), Progress in Linguistics, The Hague: Mouton, (1970) 143-173

Odegard, D.: On defining 'S knows that p', Philosophical Quarterly 15 (1965)

Partee, B. H.: Semantics- Mathematics or Psychology?, in Bauerle, R. et al. (eds.) Semantics from Different points of View, Springer Verlag (1979) 1-14

Pelletier, F. J.: The Principle of Semantic Compositionality, Topoi 13 (1994) 11-24

Sharvit, Y.: Trying to be Progressive: the Extensionality of Try. Journal of Semantics 20 (2003) 403-445

Zuber, R.: Decomposition of factives. Studies in Language 1:4 (1977) 407-421

Zuber, R.: Note on why factives cannot assert what their complement sentences express. Semantikos 4-2 (1980)

Zuber, R.: Some universal constraints on the semantic content of complex sentences. in Dirven, R. and Radden, G. (eds.) Issues in the Theory of Universal Grammar, Gunter Narr Verlag, Tubingen, (1982) 145-157

Zuber, R.: Semantic restrictions on certain complementizers. Proc. of the 12th International Congress of Linguists, Tokyo, (1983) 434-436 